\title{
EL DOGMA DE LA CALIDAD EN LA EDUCACIÓN SUPERIOR. IMPLICACIONES CONSTITUCIONALES Y FUNDAMENTACIÓN NORMATIVA EN EL ORDENAMIENTO JURÍDICO COLOMBIANO*
}

\section{The Dogma of Quality in High Education. Constitutional Implications and Normative Fundamentation in the Colombian Legal System*}

\author{
Mauricio Aragón** \\ José Luis Osorio***
}

Fecha de Recepción: Noviembre 4 de 2013

Fecha de Aceptación: Noviembre 11 de 2013

SUMARIO: 1. Introducción al problema de Investigación; 2. ¿Qué se entiende en nuestro País por Calidad en la ES?; 3. La teoría del Capital Humano; 4. La responsabilidad social universitaria; 4. Conclusiones;

\section{Referencias bibliográficas.}

\footnotetext{
"El presente Artículo de Reflexión deriva de la investigación titulada "Implicaciones teóricas de la educación en contextos de globalización" la cual se desarrolló al interior del semillero de investigación "Filosofía del derecho y derecho constitucional" en la cual los autores son los investigadores principales de la misma. Fecha de Inicio de la Investigación: 15/08/2013; Fecha de finalización: 20/10/2014.

* Abogado Egresado de la Universidad de Cartagena, miembro del Semillero de Investigaciones "Filosofía del derecho y derecho constitucional”. Email: mauricioaragon_17@hotmail.com

*** Abogado Egresado de la Universidad de Cartagena, miembro del Semillero de Investigaciones "Filosofía del derecho y derecho constitucional". Email: joseosoriogalvis@gmail.com
} 


\section{COMO SE CITA ESTE ARTÍCULO (APA 6)}

Aragón, M., \& Osorio, J. (2014) El dogma de la calidad en la Educación Superior. Implicaciones constitucionales y fundamentación normativa en el ordenamiento jurídico Colombiano. (Y. Carrillo De la rosa, Ed.) Revista Jurídica Mario Alario D'Filippo, VI (11), pág. 10-22

\section{RESUMEN}

La noción de calidad de la educación superior permea casi de manera ubicua el discurso contemporáneo en todos los aspectos del estamento universitario. El origen mercantil de tal acepción no es velado para nadie, de manera que la implementación de vocabularios propios del mundo empresarial -que proponen a la educación como un servicio, a los estudiantes como clientes, y de manera general, a la universidad como una empresa- se convierte en una realidad concretada en políticas nacionales, aterrizadas en pautas en los niveles internos de cada universidad, conformando imaginarios culturales seguidos por directivos, docentes yestudiantes.

\section{PALABRAS CLAVE}

Educación Superior, calidad, universidad, capital humano, autonomía universitaria.

\section{ABSTRAC}

The notion of quality of high education permeates almost ubiquitously the contemporary discourse on all aspects of the university status. The mercantile origin of such meaning is veiled to anybody, so that the implementation of own vocabularies of the business world - which propose to education as a service to students as customers, and generally to the university as a business- becomes a reality materialized in national policies, landed on guidelines in the internal levels of each university, shaping cultural imaginaries followed by principals, teachers and students.

\section{KEYWORDS}

Higher education, quality, university, human capital, university autonomy. 


\section{INTRODUCCIÓN AL PROBLEMA DE INVESTIGACIÓN}

Por cuenta del híper-mentado fenómeno de la globalización, el escenario universitario nacional, se somete a criterios dictados por organismos internacionales, que definen qué es y qué no es bueno en términos de educación superior, y se entiende, que la sujeción a estos preceptos posibilita la inclusión de las universidades nacionales en un mercado mundial altamente competitivo. El sistema se aferra a las pretensiones de objetividad en los estándares que miden la calidad, existe una lógica subyacente que privilegia el cumplimiento de cifras, la consecución de resultados de tipo cuantitativo, y a partir de esto se asegura el cumplimiento de estadísticas que se generan para demostrar los esfuerzos en la protección de garantías constitucionales.

A partir de la última década del siglo XX, el modelo de educación colombiano es guiado por la Constitución de 1991, y está empotrado en el Artículo 67 que consagra lo siguiente:

La educación es un derecho de la persona y un servicio público que tiene una función social; con ella se busca el acceso al conocimiento, a la ciencia, a la técnica, y a los demás bienes y valores de la cultura".

En el mismo artículo, ya en el penúltimo inciso se establece lo siguiente:

Corresponde al Estado regular y ejercer la suprema inspección y vigilancia de la educación con el fin de velar por su calidad, por el cumplimiento de sus fines y por la mejor formación moral, intelectual y física de los educandos; garantizar el adecuado cubrimiento del servicio y asegurar a los menores las condiciones necesarias para su acceso y permanencia en el sistema educativo.

Sin ninguna duda, la noción de calidad se erige como un verdadero paradigma que configura los derroteros en las políticas de educación a nivel nacional; se constituye así, una suerte de imaginario que aglutina los esfuerzos emprendidos por el Estado y que son absorbidos a su vez por la institución universitaria, convirtiéndose luego, en directrices que se imponen de manera jerárquica a los docentes encargados de transmitir ciertos saberes, estos, a la postre, deben ser asimilados por los estudiantes en su proceso de formación. "La calidad es un concepto esencial de la cultura del siglo XXI y, por tanto, conlleva a la ruptura con algunos valores tradicionales y a la aceptación de otros nuevos. La calidad en el sentido actual no es una técnica sino una filosofía." (Wesler, Adams y Bohn, 1996, citado por Lago et al., 2013)

Mirando atrás, encontramos que a finales del siglo XIX, con la Constitución de 1886, y las posteriores reformas de la primera mitad del siglo XX, el dilema principal de quienes diseñaban los modelos educativos a implementar en el país, tenía una profunda relación con luchas intestinas de tipo partidista; el quid se reducía a la participación atribuida a la Iglesia Católica en el diseño de los esquemas educacionales.

En este orden de ideas Melo (1989) comentaba que:

La historia de la educación en el siglo último muestra la íntima trabazón de las políticas 
educativas con los conflictos políticos e ideológicos del país: a partir de la Constitución de 1886, el propio canon constitucional ordena que la instrucción pública se haga de acuerdo con los principios católicos. (p.5)

Por supuesto, la influencia del catolicismo habría de determinar qué se enseñaba, cómo se enseñaba y para qué se enseñaba. En este punto, no se negociaba el contenido dogmático de dicha tradición, precisamente por la naturaleza misma de dogma.

Así como el país estuvo consagrado desde 1902 al Sagrado Corazón de Jesús con el Decreto 820 de ese año, reiterándose en ello a lo largo del siglo XX, hasta que se buscó secularizar el Estado con la Carta Política de 1991, asistimos ahora a la consagración-esta vez ideológica- del sistema de educación superior al símbolo del bien incuestionado que es la calidad.

Es primordial recordar que ya en el Decreto-Ley 80 de 1980 se venía aludiendo a esta noción, aunque no de manera tan contundente como se haría después. Lógicamente, tampoco operaba una política estatal definida en pro de la calidad en la educación. El articulado del Decreto-Ley 80 de 1980 se proponía regular la entonces llamada educación post-secundaria. De las ciento noventa y cuatro directrices que lo componían, cinco de ellas (Artículos 19, 22, 26, 52 y 140) utilizaban el vocablo "calidad" con un sentido similar al que se le atribuiría luego de 1991, esto es, entender que la calidad era una suerte de cualidad que se predicaba de la educación, una connotación que a esta se le asignaba siempre que cumpliera con los requisitos que desde el sistema y desde su escala de valores se diseñaban.

\section{2. ¿QUÉ SE ENTIENDE EN NUESTRO PAÍS POR CALIDAD EN LA ES?}

En Colombia, el Consejo Nacional de Acreditación aventuró una definición de lo que debemos entender por calidad:

El concepto de calidad aplicado al servicio público de la educación superior hace referencia a la síntesis de características que permiten reconocer un programa académico específico o una institución de terminado tipo y hacer un juicio de valor sobre la distancia relativa entre el modo como en esa institución o en ese programa académico se presta dicho servicio y el óptimo que corresponde a su naturaleza. [sic] (CNA, 2006) (Negrillas por fuera del texto original)

Estás características de las que se habla en el anterior párrafo, se trazan en el terreno del artículo 1 o del Decreto 2566 de 2003, en el que se fijaron las condiciones consideradas mínimas para atribuir el registro calificado que acredita la calidad de una institución de educación superior (IES):

Artículo 1ํ. Condiciones mínimas de calidad. Para obtener el registro calificado, las instituciones de educación superior deberán demostrar el cumplimiento de condiciones mínimas de calidad y de las características específicas de calidad. Las condiciones mínimas de calidad son las siguientes: 
1. Denominación académica del programa.

2. Justificación del programa.

3. Aspectos curriculares.

4. Organización de las actividades de formación por créditos académicos.

5. Formación investigativa.

6. Proyección social.

7. Selección y evaluación de estudiantes.

8. Personal académico.

9. Medios educativos.

10. Infraestructura.

11. Estructura académico-administrativa.

12. Autoevaluación.

13. Políticas y estrategias de seguimiento a egresados.

14. Bienestar Universitario.

15. Recursos financieros.

Las características específicas de calidad para cada programa serán fijadas por el Ministerio de Educación Nacional con el apoyo de las instituciones de educación superior, las asociaciones de facultades o profesionales o de pares académicos, siguiendo los parámetros establecidos por el Gobierno Nacional en el presente decreto.

No se evidencian con notoriedad elementos ideológicos en el contenido de los comentarios del CNA, ni en el Artículo 1ㅇ del Decreto 2566 de 2003; no obstante, se deja sentado que se debe hacer un "juicio de valor" sobre el tipo de servicio de educación que presta una institución o programa académico y el servicio que debería prestar: el óptimo que corresponde a su naturaleza. Es interesante preguntarse ¿Quién define cuál es la naturaleza de un programa académico o de una institución? Nuestra apuesta en este artículo es que, en nuestro medio, dicha definición se hace desde el mercado y para el mercado.

En el borrador de la Segunda Versión de la propuesta ACUERDO POR LO SUPERIOR (2014), desde el Consejo Nacional de Educación Superior (CESU) se escribe:

(...) la calidad de la educación superior implica un proceso sistemático y continuo de mejora sobre todos los elementos que lo integran, lo cual implica que esta sea la sumatoria de las 
partes, pues entre ellos se construyen sinergias, articulaciones y relaciones que generan un valor agregado. (p.90)

La mayoría de los discursos que ilustran las discusiones nacionales sobre el tema de la educación superior, parten de un lenguaje atravesado por nociones de tipo empresarial-insistentemente neoliberal. Basta una somera mirada a los textos que desarrollan estos debates para toparse con expresiones como costo-beneficio, valor agregado, competitividad, producción, satisfacción del cliente, control de procesos, cultura organizacional, gestión de calidad, etc.

Entre las muchísimas reflexiones que se hacen sobre calidad se escribió:

La calidad es relativa y se mide externamente como baja, media o alta, de acuerdo con el grado de satisfacción del usuario; un mismo satisfactor puede generar diferentes grados de satisfacción de acuerdo con las necesidades, sistema de valoración y expectativa de un cliente.

(Lago et al., 2009, p. 22)

Aunque ciertamente se matizan estos contenidos con alusiones a otros aspectos que informan el sistema de educación superior, y se habla de la importancia de formar mejores seres humanos, educados en valores como el de la solidaridad, la primacía de un itinerario empresarial se observa de golpe. En la puja entre los valores materialistas y deshumanizados del mercado neoliberal, y los valores de fraternidad y solidaridad que se supone modulan nuestro ordenamiento jurídico, es tristemente obvio que ganan los más perniciosos y dañinos.

En un reciente informe para el Ministerio de Educación Nacional entregado por el experto internacional en reformas para la educación superior, Jamil Salmi, el autor citaba como epígrafe en la introducción de su trabajo, una frase del premio Nobel de economía Paul Krugman: La productividad no es todo, pero a largo plazo es casi todo. Este tipo de análisis sin duda se desarrollan al tenor de una visión neoliberal, en donde "(...) todas las transacciones humanas se consideran como formas de intercambio económico." (Smith, 2004, p. 118) Precisamente Smith (2004) citando a Cope y l'Anson escribe:

(...) "dentro de este proyecto, la educación ha sido discursivamente reestructurada de acuerdo con la lógica del mercado, y con la política educativa cada vez más colonizada por iniciativas de política económica", con la educación consecuentemente transformada en la adquisición de conocimiento instrumental y habilidades comercializables.

La consabida desconfianza que desde el neoliberalismo se proyecta hacia lo público (El Estado es mal empresario) no solamente se refleja en las agresivas políticas de privatización, sino también en esos contenidos lingüísticos que hoy dominan las discusiones sobre el sistema de educación superior. López Rupérez ha dicho (como se citó en Viñao Frago, 2001) “(...) cabe otorgar a la noción de privatización un significado "blando", consistente en la implantación en el ámbito de lo público de los procedimientos que se han revelado eficientes en la gestión de lo privado".

Estas palmarias muestras de mercantilismo académico han posibilitado espacios de reflexión en los que se asevera, como lo hizo Viñao Frago (2001) en el IV Encuentro Regional de la Asociación para la 
Mejora y Defensa de la Escuela Pública en la Región de Murcia:

La calidad deviene, de este modo, algo cuya responsabilidad corresponde, en exclusiva, a los centros docentes, es decir, a los profesores, a los padres y, sobre todo, a la dirección del centro docente. El director se convierte en un manager o gerente, cuyo éxito se mide comparando los resultados académicos de su centro con los de otros y, sobre todo, con la mayor o menor demanda o cuota de mercado que se alcanza. El centro docente, con independencia de su titularidad pública o privada, pasa a ser considerado como una empresa en un mercado en el que compiten los centros privados con los públicos y todos ellos entre sí, y en el que los mejor gestionados son, teóricamente al menos, los más demandados (o en el que, si se prefiere, los más demandados son los mejor gestionados y los que mejores resultados obtienen). (p.6,7)

La situación que atraviesa la educación superior en Colombia se enmarca dentro de una serie de ajustes estructurales que han sido iniciados más o menos desde que arrancó entre nosotros la apertura económica. La ostensible implementación de políticas neoliberales no sucede únicamente en el campo educativo.

De manera sucinta, Bidaurrazaga (como se citó en Honrubia, 2009) escribe del neoliberalismo que:

Sus propuestas de organización de la actividad económica en su lucha en favor del equilibrio macroeconómico pueden ser resumidas bajo la fórmula "más mercado, menos Estado", dando origen al proceso de desregulación, privatizaciones, reducción de la protección social, precarización laboral y, en definitiva, de desestructuración del Estado de Bienestar característico de las economías capitalistas industrializadas durante las décadas que duró la hegemonía keynesiana.

En el caso colombiano, aun con la Ley 30 de 1992 que en su momento fuera recibida en medio de aplausos y que suponía medidas de apoyo financiero dentro de los márgenes de una noción de autonomía que fue percibida con esperanza, las circunstancias de financiación de la universidad pública no arrojan a estas alturas un panorama saludable. En el Informe de Educación Superior en Iberoamérica para el 2011 presentado por el Centro Interuniversitario de Desarrollo (CINDA) se dice lo siguiente:

"Durante el último quinquenio, no se produjo ningún cambio significativo en el financiamiento de la educación superior, a pesar de que la cobertura se incrementa. Las universidades públicas siguen financiadas con base en la Ley 30 de 1992, que define la política de financiación del sistema estatal de universidades. Los aportes otorgados bajo esta ley se ajustan con base en la inflación, por lo que en términos constantes reciben casi los mismos recursos. Sin embargo, como proporción del PIB estos disminuyen ya que al comienzo del período representaron el 0,50 y al final el 0,47 . (p.309)

No puede perderse de vista que se trata de una actitud general hacía las políticas sociales, en donde la mercantilización y las des-estatización comprometen la mayoría de las esferas de la sociedad. La tesis aplicada para los años 80 s proponía que siendo que las dificultades institucionales de la universidad eran insuperables se "imponía" la salida de convertir ese bien público en un mercado. A continuación serían dos los procesos que articularían el proyecto neoliberal con la educación: la 
descapitalización de la universidad pública y la globalización mercantil de la misma. Este último proceso, o sea, la mercantilización de la universidad, opera en dos niveles. Desde el primero se busca llevar a la universidad a la consecución de sus propias fuentes de financiación para afrontar la crisis económica. El segundo de los niveles mencionados supondrá el desdibujamiento constante entre universidad pública y privada. Sin esta distinción se irá convirtiendo en una empresa en cuanto a sus relaciones externas, y además, la lógica de sus dinámicas internas deberá ser la misma, esto es, operar con criterios de mercado. (de Sousa Santos, 2009) El adoptar una postura crítica ante estos asuntos implica el reconocer cuál es el origen de las políticas que se vienen implementando en la región: "Se trata de un proceso global y es esa la escala en que debe ser analizado." (de Sousa Santos, 2009, p. 17)

Para que una nación en vías de desarrollo se haga beneficiaria de un préstamo del Banco Mundial o del Fondo Monetario Internacional, debe atender las recomendaciones que hagan estas organizaciones para así recibir la ayuda necesitada. En general, se propende por una progresiva desregulación de los sistemas bancarios, liberalización de los precios, austeridad presupuestaria, entre otras medidas. Sobre la política del BM a propósito de la educación superior, de Sousa Santos (2009) anota lo siguiente:

La posición del Banco Mundial en el área de la educación es tal vez de las más ideológicas que este ha asumido en la última década (y no han sido pocas) porque tratándose de un área donde todavía dominan interacciones no mercantiles, la embestida no puede basarse en un mero lenguaje técnico como el que impone el ajuste estructural. La inculcación ideológica se sirve de análisis sistemáticamente sesgados contra la educación pública para demostrar que la educación es potencialmente una mercancía como cualquiera otra y que su conversión en mercancía educativa explica la doble constatación de superioridad del capitalismo en cuanto organizador de las relaciones sociales y de la superioridad de los principios de la economía neoliberal para maximizar las potencialidades del capitalismo a través de la privatización, desregulación, mercantilización y globalización. [sic] (p.22)

Tal parece que los postulados que se defienden desde el pensamiento neoliberal están "inscritos en un código genético de la sociedad". Santos Guerra (1999) comenta que la competitividad y la eficacia son señuelos que sin duda condicionan culturalmente a los individuos insertos en este modelo. Este autor español, citando a Le Mouël escribe que, "La eficacia, como sinónimo de la calidad, es el mito contemporáneo". Luego agrega que la educación “(...) es arrastrada por tesis aparentemente incontrovertibles". (Santos Guerra, 1999, p.78)

\section{LA TEORÍA DEL CAPITAL HUMANO}

Desde los años 80s se empieza a considerar que en vista de que la educación implica un costo económico para los países, esta debe redituar en desarrollo, de no hacerlo, podía empezar a hablarse de una crisis en el sistema educativo. Según estos planteamientos, la educación debe ser mirada como una inversión. Como expresa Bianchetti (2009), la educación como inversión es un principio que se proyecta en un rango de análisis económico macro y en uno micro:

El primero, estudia la incidencia que ejerce sobre la renta nacional, el aumento de los costes 
públicos y privados en educación. El segundo, estudia, cómo la mayor acumulación de años de educación a nivel individual, dará lugar a un incremento de la productividad futura del trabajador y por lo tanto un aumento de sus ingresos.

Ambos abordajes fueron utilizados por parte de investigadores, gobiernos y organismos internacionales, con el fin de evaluar la relación "costo - beneficio", tanto a nivel individual, como también en relación con los índices que expresan el crecimiento económico.

Estos análisis contienen una escala de valores, que sirve para establecer prioridades en la toma de decisiones de Política Educacional y son además, las que le otorgan el sentido originario al concepto "calidad". (p.9)

Por su origen mismo, el concepto de calidad lleva implícita la posibilidad de ser medido, de ser cuantificado, es así que se facilita el proceso de comparación y de competencia entre productos. Para los años 80 s, la percepción más extendida era que una persona tenía un mejor nivel de educación en tanto tuviese mayor número de años de educación individual. A su vez se pensaba, que el sistema estaba en mejores condiciones si las tasas de matrículas eran mayores a las de otras naciones, además de que también lo fuesen las tasas de graduación. La acumulación de años de escolaridad se mantenía gravitando en torno al principio de la educación como inversión. En todo caso, se trataba de establecer criterios de cuantificación, y estos que se mencionan se tornaron insuficientes cuando los países-potencia, fueron capaces de masificar su oferta educativa. ¿Cómo medir entonces si todos los alumnos tenían un similar número de años de escolaridad? La noción del capital humano ya no fue adecuada para explicar las diferencias de competitividad entre las naciones, de manera que se dirigió la mirada hacia criterios cualitativos, esto implicaba analizar el sistema internamente, de lo demográfico a los contenidos mismos de la enseñanza. En palabras de Juan Casassus, quien para 1999 era especialista regional de la UNESCO en estos temas:

En una primera instancia se procedió a la elaboración de hipótesis acerca de los factores "materiales" que determinan el resultado. Surgieron elementos tales como las razones de relación profesor/alumno; número de libros en casa o en las escuelas, la luminosidad en el aula - la cantidad de alumnos en ella. Más tarde se formularon hipótesis acerca de factores "inmateriales", como son las expectativas y las interacciones que se dan al interior de las escuelas o en el hogar. Pero, finalmente, la atención ha terminado por fijarse en su foco actual; la observación y medición del logro académico. (Cassasus, 1999, p. 49)

Y más adelante aclara por completo el panorama:

El proceso de medición es en esencia simple: normalmente se trata de elaborar pruebas de logros esperados, las que son aplicadas a los alumnos. En este contexto, el concepto "calidad" adquiere una definición operacional muy precisa: calidad es el porcentaje de logro de los objetivos educativos. En este lenguaje, el objetivo mismo no es cuestionado. Se da por supuesto que el objetivo a medir corresponde a lo que los estudiantes deben aprender. (Cassasus, 1999, p. 49) (Negrillas fuera del texto original)

En realidad el texto citado está cargo de falacias de argumentación, pero nos interesa concretamente la que está evidente en el párrafo transcrito. La "petición de principio" usada, revela 
que la espina dorsal del concepto de calidad no es otra cosa que una narrativa, una serie de valores escogidos para fungir como verificables, una ideología es la que repta en estos conceptos. Unas cuantas líneas más adelante el autor explica: "Más aún, es posible postular que la fuerza del concepto de calidad radica precisamente en su ambigüedad." (Cassasus, 1999, p. 50)

\section{LA RESPONSABILIDAD SOCIAL UNIVERSITARIA}

En 1841 Edgar Allan Poe publicó un relato titulado The Murders in the Rue Morgue (Los asesinatos de la calle Morgue) el narrador, describe las hazañas intelectuales de August Dupin, quien lograría descifrar con éxito un crimen misterioso que no había sido resuelto por el prefecto de policía encargado de la investigación. Al final del relato, Dupin explica que la causa del fracaso en los esfuerzos del prefecto radicó en su exceso de razón. Su método era comparado con la diosa Laverna, deidad romana de los ladrones representada como una mujer con una enorme cabeza y un cuerpo desproporcionadamente pequeño. A través de esta metáfora Poe elevaba en ese contexto, una de las primeras críticas que desde la literatura se hacían a la ciencia cartesiana. Imaginemos por un momento a esta ciencia tratando de avanzar en el camino de la historia sosteniendo esa enorme cabeza llena de números y abstracciones. El prefecto de policía aun habiendo sido derrotado seguía manteniendo su vanidad y autosuficiencia ampulosa, pese a haberse demostrado su incapacidad para resolver el problema. De él, como representante de la ciencia de la época, Dupin decía que, debía su reputación de hombre talento al hecho de de nier ce qui est, et d'expliquer ce qui n'est pas (De negar lo que es, y explicar lo que no es)

La obsesión por el número y la abstracción, característica de las sociedades contemporáneas, ha sido ampliamente denunciada y retratada en sus peligros. En su artículo "Abstraction and finitude: education, chance and democracy" (Abstracción y finitud: educación, azar y democracia) el filósofo de la educación Richard Smith (2004), comenta la crisis que atraviesa la educación superior por cuenta del paradigma de una cultura calculadora, utilitaria, y entregada a "rituales de verificación" al tiempo que se deshumaniza privilegiando un discurso económico.

La incursión de la nueva concepción de autonomía que se consagró en la Constitución de 1991, y su desarrollo legal contenido en la Ley 30 de 1992, atrajo hacía las universidades públicas la atención de la sociedad, acarreando mayores compromisos de autoevaluación si se querían alcanzar los recursos que el Estado pudiera entregar, una vez demostrado el cumplimiento de las metas propuestas. Esta responsabilidad social que se está exigiendo no es llana filantropía; estando las IES empapadas hasta el tuétano de empresarismo, se busca cuantificar al tenor que impone el modelo, dicha responsabilidad, que viene a ser entendida como "valor agregado" y como sostenibilidad económica.

En el artículo titulado Educación para un nuevo humanismo, el filósofo colombiano Guillermo Hoyos (2009) sostiene que "(...) parece que el indicador reina de la responsabilidad social universitaria, y en general de la educación es pertinencia" (p. 427). Y es que dentro del desarrollo conceptual de la noción de calidad, este es uno de los aspectos más renombrados y discutidos. Para José Silvio (2006) la pertinencia "Consiste en la adecuación de la educación al contexto social en el cual se desempeña la persona, en el ámbito grupal, organizacional, comunitario y social." (p.10) 
En el marco de la Ley 30 de 1992, Lago et al. (2009) citando a Ossa ha escrito sobre este tópico que:

La pertinencia de la educación se refiere al grado en que esta contribuye al logro de determinados objetivos sociales y económicos, definidos como prioritarios, estratégicos o deseables en la sociedad. La autonomía otorgada por la Ley 30 de 1992, en Colombia, es una herramienta fundamental para que responsablemente las instituciones de educación superior, ofrezcan educación pertinente, esto es, que obedezca a criterios sociales, y no a aquellos determinados únicamente por la sola fuerza del mercado o por las estrategias referidas de cada institución. (p.20)

Sobre este particular, el Consejo Nacional de Educación Superior expresó:

Desde una perspectiva política, la noción de pertinencia se refiere al papel desempeñado por la educación superior como sistema, y por cada una de sus instituciones y organizaciones con respecto a la sociedad, igualmente lo que el entramado social espera (tasa de retorno) del sistema de educación superior. (CESU, 2014, p.150)

He aquí uno de los momentos de mayor dificultad de equilibrio entre dos postulados ineludibles en estos debates. Hasta este punto ha quedado dicho que desde que el país se resguarda en la Constitución de 1991, y con ocasión de la Ley 30 de 1992, quienes reflexionan sobre estos temas, encumbran la autonomía como una gran conquista al comparársela con la experiencia acaecida bajo la legislación anterior. Además, se subraya que el papel del Estado no viene hacer policivo sino garante. Entonces, bajo estas nuevas "gabelas", la universidad debió asumir obligaciones con la sociedad a la cual pertenece. "Del mismo modo, la mayor autonomía que fue concedida a las universidades no tuvo como objetivo preservar la libertad académica, sino crear condiciones para que las universidades se adaptaran a las exigencias de la economía." (de Sousa, 2009, p. 18, 19)

Para aterrizar los comentarios citados arriba, ya en la realidad nacional esbocemos algunas reflexiones en torno a la Ley 1286 de 2009 que modificó la Ley 29 de 1990, y que vino a transformar a Colciencias en Departamento Administrativo. El Artículo primero de esta normativa reza en el siguiente tenor:

Artículo; $1^{\circ}$. Objetivo general. El objetivo general de la presente ley es fortalecer el Sistema Nacional de Ciencia y Tecnología y a Colciencias para lograr un modelo productivo sustentado en la ciencia, la tecnología y la innovación, para darle valor agregado a los productos y servicios de nuestra economía y propiciar el desarrollo productivo y una nueva industria nacional.

He aquí el enfoque actual de la investigación en nuestro país. La propensión más evidente retratada a lo largo de esta legislación está casada con un esquema para el que la búsqueda de conocimientos debe estar al servicio de la industria como condición necesaria del desarrollo económico. Además, se propugna por insertar el sistema colombiano de investigación en un contexto internacional que opaca fronteras nacionales. Más adelante volveremos sobre la necesidad de afianzar el proyecto de nación, aclarando que no se trata de uno autárquico ni nacionalista.

El Artículo 6ㅇ, contentivo de los objetivos generales de Colciencias consagra que: 
3. Fundamentar y favorecer la proyección e inserción estratégica de Colombia en las dinámicas del sistema internacional que incorporan el conocimiento y la innovación y generan posibilidades y desafíos emergentes para el desarrollo de los países y sus relaciones internacionales, en el marco de la sociedad global del conocimiento.

Por supuesto que la comprensión de las actuales circunstancias que rodean a la universidad, determina que esta se amolde a dinámicas de internacionalización, pero percatarse de la forma en la que viene volcándose fuera de las fronteras nacionales sin una atinada definición de sus nortes, al margen de procesos puramente neoliberales, es lo que debe entrar a corregirse.

La universidad es un bien público íntimamente ligado al proyecto de nación. El sentido público y cultural de este proyecto y su viabilidad, dependen de la capacidad nacional para negociar de manera calificada, la inserción de la universidad en los contextos de transnacionalización. En el caso de la universidad y de la educación en general, esa cualificación es la condición necesaria para no transformar la negociación en un acto de rendición y con él, el fin de la universidad tal como la conocemos. No habrá rendición si existen condiciones para una globalización solidaria y cooperativa de la universidad. (de Sousa, 2007, p. 68)

Preocupa saber cuál es el papel que la Ley 1286 de 2009 da a la investigación en las ciencias sociales y humanas. Sobre esto, el numeral 7으 del Artículo 6올ala:

7. Integrar esfuerzos de los diversos sectores y actores para impulsar áreas de conocimiento estratégicas para el desarrollo del país, en las ciencias básicas, sociales y humanas, de acuerdo con las prioridades definidas en el Plan Nacional de Desarrollo.

Yéndonos entonces a la letra del PND del año 2010 encontramos lo siguiente:

"Los sectores agropecuario, minero-energético, infraestructura de transporte (...) deberán definir en el 2011 cuáles son sus necesidades de recurso humano (en términos de perfiles, competencias y programas de formación requeridos) de tal manera que el MEN, como regulador de la formación del capital humano, la capacitación y el aprendizaje, genere los incentivos a las instituciones de educación para el trabajo y el desarrollo humano, para PRIORIZAR la formación y el desarrollo de las competencias laborales específicas de acuerdo a las necesidades definidas por estos sectores". (Bases del PND Colombia, 2010)

Al respecto de la investigación social en la normativa citada, el profesor Hoyos comenta no sin sarcasmo que, se la está archivando en un "cuarto de San Alejo". Ello explica el que haya expresado que "(...) la concepción de la ciencia y la tecnología en Colombia sigue siendo reduccionista en el sentido más tradicional del positivismo científico." (Hoyos, 2008, p.428)

\section{CONCLUSIONES}

Los contenidos ideológicos que se despliegan sobre el sistema de educación colombiano bajo el amparo de la Carta Constitucional, no surgen de la cuasi omnipresente expresión "calidad". El modificar este término por supuesto que no implica una mejoría instantánea del problema que padece nuestro modelo. Lo que hemos querido denunciar es que la estructura axiológica que se defiende a partir de la noción de calidad, y la historia y el contexto que esta implica, resultan 
perniciosas en un país como el nuestro. Enaltecer los valores de la competencia "predatoria" que en la nación se toman como norte, supone según quisimos demostrar, un arma de doble filo. Siendo que lo que nos une como colombianos es una historia de tragedias comunes, de heridas que no han logrado sanar, no parecería sensato que se traten de sobrellevar los dilemas del país, incentivando entre los ciudadanos la búsqueda de la mejoría por sobre las posibilidades de avance para el otro. Esta reflexión se aplica en el escenario de las competencias entre universidades, entre programas académicos, entre profesores, entre estudiantes. Si bien nuestro ordenamiento habla de solidaridad, una mirada incluso somera sobre el sistema revela que tal solidaridad no es más que un enunciado, pero que en el plano de la acción estamos lejos de desarrollos basados en tal principio.

\section{REFERENCIAS BIBLIOGRÁFICAS}

BIANCHETTI, G. (2009) "Educación de calidad: uno de los dilemas fundamentales para las políticas educativas que se propongan demostrar, que 'otro mundo es posible'".

CASSASUS, J. (2009) "Lenguaje, poder y calidad de la educación". Boletín 50, diciembre 1999 / Proyecto principal de educación.

CENTRO INTERUNIVERSITARIO DE DESARROLLO (CINDA) "Informe de Educación Superior en Iberoamérica para el 2011".

\section{CONSEJO NACIONAL DE ACREDITACIÓN (CNA) 2006.}

CONSEJO NACIONAL DE EDUCACIÓN SUPERIOR (CESU) (2014) "Borrador de la Segunda Versión de la propuesta ACUERDO POR LOSUPERIOR".

DE SOUSA, B. (2005) "La universidad en el siglo XXI. Para una reforma democrática y emancipadora de la universidad". Revista Umbrales

HONRUBIA, P. (2009) “Neoliberalismo y desarrollo, la historia de un fracaso anunciado”.

HOYOS, G. (2009) “Educación para un nuevo humanismo". Magis Revista Internacional de investigación en Educación, 2, 425-433.

LAGO, D., y LÓPEZ, E., MUNICIO, P., OSPINA, R., VERGARA, G. (2013) “La calidad de la educación superior: ¿un reto o una utopía?" Ecoe ediciones: Universidad de Cartagena.

MELO, J. (1989) "Educación y ciencia". En Educación y ciencia: Luchas de la mujer. Vida diaria (Ed) Nueva Historia de Colombia Tomo. IV (5-7) Bogotá, Colombia: Planeta Colombiana Editorial. 1989.

SALMI, J. (2013) "La Urgencia de Ir Adelante: Perspectivas desde la Experiencia Internacional para la Transformación de la Educación Superior en Colombia”.

SANTOS, M. (1999) “Las Trampas de la Calidad” Revista Acción pedagógica a, vol. 8, no. 2/1999.

SILVIO, J. (2006) "Hacia una educación virtual de calidad, pero con equidad y pertinencia". Revista de Universidad y Sociedad del Conocimiento Vol. 3-N. o1/Abril de 2006.

SMITH, R. (2006) “Abstracción y finitud: Educación, azar y democracia”. Revista Devenires VII, Vol. 13 p. 112131.

VIÑAO, A. (2001) “El concepto neoliberal de calidad de la enseñanza: Su aplicación en España (1996-1999)" D.L. MU 783-2001 ISSN 1577-7995. 